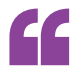

patient iMNs

had fewer

lysosomes

than did

controls, indicating a

disruption

of lysosome

biogenesis in

the patients

NEURODEGENERATIVE DISEASE

\title{
C9orf72 insufficiency cooperates with toxic repeat peptides to kill neurons
}

A new study has shed light on how the most common known cause of amyotrophic lateral sclerosis (ALS) and frontotemporal dementia (FTD) - a GGGGCC-repeat expansion in C9orf72 - exerts its toxic effect and leads to neuronal degeneration. Investigators found that a loss of C9orf72 protein disrupts endosomal trafficking, resulting in an accumulation of glutamate receptors and defective breakdown of toxic dipeptide-repeat (DPR) proteins, both of which promote the death of motor neurons.

The repeat expansion in C9orf 72 causes several problems in cells, including decreased levels of native C9orf72 and the accumulation of RNA and DPR proteins manufactured from the repeats. However, how these different effects combine to bring about ALS and/or FTD in patients is uncertain, which poses a challenge for the development of therapies for individuals with this mutation.

In the new report, Justin Ichida and colleagues studied induced motor neurons (iMNs) generated from patients with the repeat expansion (patient iMNs) and from healthy individuals without the expansion (control iMNs). Patient iMNs had lower levels of C9orf72 than did control iMNs and underwent rapid neurodegeneration when treated with glutamate. The same defect was observed in control iMNs treated with an antisense oligonucleotide against C9orf72. The heightened glutamate sensitivity was associated with an increased number of NMDA and AMPA receptors on the cell surface of C9orf72-deficient iMNs and was reversed by overexpression of C9orf72.

To see how loss of C9orf72 might exert this excitotoxic effect, the team assessed the location of the protein and found that it colocalizes with markers of early endosomes in control and patient iMNs. However, patient iMNs had fewer lysosomes than did controls, indicating a disruption of lysosome biogenesis in the patients. Conversely, overexpression of $\mathrm{C} 9$ orf 72 restored the number of lysosomes.

"We wondered whether reduced C9orf72 activity renders motor neurons vulnerable to other insults that might be exacerbated by endosomallysosomal system function, such as
DPR toxicity," Ichida and colleagues write. When the researchers overexpressed C9orf72 in patient iMNs, they found that it reduced the number of aggregates that contain the toxic DPR protein proline-arginine.

Interestingly, the team also identified several small molecules that improved the survival of patient iMNs by modulating endosomal trafficking pathways, suggesting that these processes might be a therapeutic target in individuals with ALS or FTD who have the C9orf72 repeat expansion.

The investigators claim that their findings highlight the relevance of both loss-of-function and gain-offunction mechanisms of pathogenesis in patients with the C9orf 72 repeat expansion. "Our results establish a new approach for suppressing DPR protein toxicity and blocking C9orf72 pathogenesis: restoring or replacing C9orf72 activity," Ichida and colleagues write.

Charlotte Ridler

ORIGINAL ARTICLE Shi, Y. et al.

Haploinsufficiency leads to neurodegeneration in

C9ORF72 ALS/FTD human induced motor neurons.

Nat. Med. https://doi.org/10.1038/nm.4490 (2018) 